\title{
Trojan Horse Strategies Used by Pathogens to Influence the Small Ubiquitin-Like Modifier (SUMO) System of Host Eukaryotic Cells
}

\author{
Miklós Békés ${ }^{a} \quad$ Marcin Drag ${ }^{b}$ \\ a Department of Biochemistry, New York University School of Medicine, New York, N.Y., USA; ${ }^{b}$ Division of Medicinal \\ Chemistry and Microbiology, Faculty of Chemistry, Wroclaw University of Technology, Wroclaw, Poland
}

\section{Key Words}

Small ubiquitin-like modifier $\cdot$ Protease $\cdot$ Host cells •

Pathogenic bacteria and viruses

\begin{abstract}
A remarkable feature of pathogenic organisms is their ability to utilize the cellular machinery of host cells to their advantage in facilitating their survival and propagation. Posttranslational modification of proteins offers a quick way to achieve changes in the localization, binding partners or functions of a target protein. It is no surprise then that pathogens have evolved multiple ways to interfere with host posttranslational modifications and hijack them for their own purposes. Recently, modification of proteins by small ubiquitin-like modifier has emerged as an important posttranslational modification regulating transcription, DNA repair and cell division, and literature has started to emerge documenting how it could be utilized by pathogenic bacteria and viruses during infection. In this brief review, we focus on the host small ubiquitin-like modifier (SUMO) system and how disease causing agents influence SUMO conjugation and deconjugation, highlighting the common theme of global hypoSUMOylation upon infection by pathogens.
\end{abstract}

Copyright $\odot 2012$ S. Karger AG, Basel

\section{KARGER}

Fax +41613061234 E-Mail karger@karger.ch www.karger.com
(C) 2012 S. Karger AG, Basel

$1662-811 X / 12 / 0042-0159 \$ 38.00 / 0$

Accessible online at:

www.karger.com/jin

\section{Introduction}

Posttranslational modification (PTM) of cellular proteins is a widespread biological mechanism that encompasses multiple strategies, which have evolved since early prokaryotes to modify the activity, subcellular localization or binding partners of the modified proteins. Certain types of PTM, such as glycosylation, acetylation, phosphorylation and the proteolytic processing of substrates are highly conserved between prokaryotes and eukaryotes, while others, such as modification by ubiquitin and ubiquitin-like molecules are found mostly in eukaryotic organisms [1], with the recently discovered exception; Mycobacterium tuberculosis has been shown to harbor a prokaryotic ubiquitin-like protein (UBL) - Pup which is also covalently attached to its targets and plays a role in the proteasome pathway of the bacterium [2].

Posttranslational modifications, other than the proteolytic cleavage of a target protein, are reversible and highly dynamic, whether employed by prokaryotes or eukaryotes. PTMs can be added to target proteins as well as rapidly removed by distinct enzymatic cascades and by editing enzymes, respectively. Just as phosphorylation by kinases is counteracted by phosphatases [3], acetylation is reversed by deacetylation [4] and N-glycosylation is removed by the action of glucosidases [5]. Ubiquitination 
(the addition of ubiquitin molecules) is balanced by the action of deubiquitinating enzymes (DUBs) that cleave ubiquitin from conjugated proteins [6]. Accordingly, SUMOylation - modification of proteins by SUMO (small ubiqutin-like modifier) [7] - in eukaryotes is negated by the action of deSUMOylating proteases [8], while PUPylation in $M$. tuberculosis has also been shown to be reversed by dePUPylation by the isoproteolytic cleavage of the conjugated Pup-molecule [9].

Interestingly, while across taxa PTMs serve in housekeeping mechanisms that play roles in cellular homeostasis and signaling cascades, various pathogens can also utilize PTM mechanisms to their advantage in ensuring the effective infection of host organisms. For example, viruses have long been known to take advantage of cellular PTM strategies, such as the ubiquitin-proteasome pathway $[10,11]$ or utilizing host phosphorylation/dephosphorylation dynamics $[12,13]$, as well as influencing host proteolytic cascades by delivering their own protease inhibitors, such as the viral serpin CrmA, to inhibit caspases and to prevent the cell death response of the host cell $[14,15]$. Bacterial pathogens have also developed a number of ways to modulate host PTM mechanisms, excellently reviewed by Ribet and Cossart [16], providing a highly useful overview of PTMs that are hijacked by bacteria during various stages of infection. In this review, we will focus on the deSUMOylation dynamics of eukaryotic host cells and how they are directly and indirectly affected during infection by bacterial and viral pathogens.

\section{The SUMO System}

SUMO was discovered some 15 years ago as a covalent modifier of RanGAP1 [17] and has since emerged as an important PTM, regulating a plethora of biological processes, such as transcription [18], ribosome biogenesis [19], receptor function [20], cell cycle control [21] and DNA repair $[22,23]$. There are three SUMO molecules in humans, SUMO1, SUMO2 and SUMO3. SUMO2 and SUMO3 are more than $90 \%$ identical to each other and are commonly referred to as SUMO2/3; however, they are only 50\% identical to SUMO1 [24]. The differences between SUMO paralogs provides specificity for SUMO precursor activation by the proteases that cleave them [25] and are important in directing binding specificity toward proteins with SUMO-interacting motifs [26]. SUMO2 and SUMO3 also form chains both in vitro and in vivo $[27,28]$. PolySUMOylation utilizes conserved ly- sine residues that are found in the $\mathrm{N}$-terminal regions of SUMO2 and SUMO3, but are not found in SUMO1. It is a highly dynamic process which is held at bay by the proteases that remove SUMO [29] and has been shown to be influenced by various exogenous stress stimuli [30, 31]. In addition, it can serve as an indirect degradation signal, as, upon polySUMOylation, some polySUMOylated proteins have been shown to be ubiquitinated by polySUMOylated protein-specific ubiquitin E3 ligases and subsequently degraded in a proteasome-dependent manner [32-34].

SUMOylation - the covalent attachment of a SUMO molecule to a lysine side-chain of a target protein (see fig. 1) - is an energy-dependent process that is analogous to the conjugation of various UBLs, albeit with specific conjugating enyzmes for each ubiquitin-like molecule [24]. Among these various UBLs, their ligation pathway is conserved and so is their removal (deconjugation) carried out by specific proteolytic enzymes. Prior to conjugation, SUMO precursors expressed with C-terminal tails after the conserved glycine residue required for conjugation, also have to be processed by the aforementioned proteases, called sentrin-specific proteases (SENPs) [35].

SUMOylation of a target protein happens within a loosely defined consensus sequence, $\psi \mathrm{KXE}$ (where $\psi$ is a hydrophobic amino acid, $\mathrm{K}$ is lysine, $\mathrm{X}$ is any amino acid and $\mathrm{E}$ is glutamic acid), where the $\varepsilon$-amine of the lysine is modified through an isopeptide-bond via the carboxylate of the C-terminal glycine of SUMO [36, 37]. The conjugation arm of the SUMOylation pathway requires an E1 SUMO-activating enzyme [38], which activates the processed SUMO molecule by an elegant adenylation mechanism [39, 40]; an E2-conjugating enzyme, which transfers the activated SUMO molecule onto its active site cysteine [41], and in some cases an E3 ligase. There is a single active E1 in humans and in yeast, the Asol-Uba2 heterodimer [38], and a single active E2, Ubc9 [41]. Ubc9 is capable of directly SUMOylating target proteins in vitro [42]; however, in vivo the specificity of SUMOylation is likely conferred by a handful of SUMO E3 ligases [43]. But the SUMO cycle is far less complex than the attachment of ubiquitin, which utilizes multiple E2s [44] and hundreds of E3s [45], making the SUMO system fairly susceptible to interference by pathogens. For example, the adenoviral protein Gaml has been shown to inhibit the SUMO E1 enzyme and cause global host deSUMOylation [46], and recently a bacterial virulence factor, listeriolysin O (LLO), has also been implicated in causing global deSUMOylation by the degradation of the sole SUMO E2 enzyme, Ubc9 [47]. Details on how infectious 
agents interfere with the SUMO system are discussed below.

Finally, the SUMO cycle is concluded with the recycling of the SUMO molecule and the lysine of the target protein by the proteolytic cleavage of the isopeptide-bond by SENPs. Such deSUMOylation activity is again less complex than deubiquitinylation, which is carried out by approximately 100 DUBs, which span several protease families $[6,48]$. On the other hand, in humans deSUMOylation is executed by 6 SUMO-specific proteases that begin and end the SUMO cycle [8].

\section{DeSUMOylating Proteases}

DeSUMOylating enzymes (SENPs) are cysteine proteases that belong to peptidase family $\mathrm{C} 48$, based on sequence and structural classification according to the MEROPS database [49]. Ulp1 and Ulp2, the first SENPs identified [50, 51], were discovered in Saccharomyces cerevisiae as proteases that cleave Smt3, the yeast SUMO molecule, but not ubiquitin [52]. Later, SENPs were found and characterized in organisms throughout taxa, in parasites such as Pf-SENP1 in Plasmodium falciparum [53] and (importantly, for the purpose of this review) in bacteria, such as XopD, which was found in a phytopathogenic bacterium, Xanthomonas campestris pathovar vesicatoria [54]. In humans, there are 7 fully functional SENPs numbered 1-8 with the exception of SENP4, a pseudogene [35].

SENPs are dual-action proteases and are responsible for two distinct activities on SUMO proteins which are mechanistically identical, but functionally different. First, they activate SUMO precursors by cleaving their C-terminus to expose the glycine required for conjugation (endopeptidase activity). Second, they deconjugate SUMO from its targets (isopeptidase activity). Substrate specificity analysis reveals that SENPs are one of the most specific proteases and recognize only SUMO molecules as their substrates [25], with the exception of SENP8, which recognizes and cleaves another ubiquitin-related modifier, Nedd8 [55]. For an in-depth description of SENPs, please refer to recent reviews which focus intensively on their catalytic properties $[35,56,57]$. We briefly discuss some of their essential properties.

The mechanism of SUMO recognition by SENPs involves two important regions within their catalytic domains. One of these regions, called the exosite, forms most of the contacts with the SUMO core and is crucial for binding and activity $[25,58]$. The other region re-



Fig. 1. SUMOylation/deSUMOylation dynamics: the SUMO pathway in eukaryotes, depicting SUMO activation by E1, SUMO ligation by the E2 and E3 enzymes onto target proteins, and subsequent SUMO deconjugation by SUMO proteases, which also process SUMO to its active form.

quired for SUMO recognition is the classic peptide sequence next to the active site, which is responsible for correctly aligning the C-terminal tail of SUMO in the active site of the protease $[59,60]$. In principle, the mechanism of SUMO recognition by SENPs is very similar to that of DUBs recognizing ubiquitin, with the notable exception that SENPs do not undergo conformational changes upon substrate binding as do some DUBs $[6,61]$.

Besides the specificity of SENPs toward SUMO molecules, human SENPs also exhibit specificity for different SUMO paralogs. SUMO1 is cleaved by SENPs 1 and 2, while SUMO2/3 is cleaved by all SENPs with varying activities. However, there is a clear difference for SENPs 6 and 7 , which are practically inactive as endopeptidases towards SUMO precursors and exhibit only modest activity towards the model substrate RanGAP1, conjugated by SUMO paralogs. However, it has been shown that SENPs 6 and 7 are very effective in processing polySUMO chains [62]. A recent finding, using SENPs 1 and 6, also demonstrated that all SENPs can actually recognize and process polySUMO chains equally well [29], showing that SUMO chain deconjugation is one of their intrinsic properties.

Phylogenetic analysis of human SENPs separates them into 4 groups: SENPs 1 and 2, SENPs 3 and 5, SENPs 6 and 7 and SENP8. Combinatorial library screening using tetrapeptide sequences as substrates confirmed this clus- 
tering experimentally. It has been determined that SENPs 1,2 and 5 preferentially recognize substrates based on the sequence of the native C-terminus of SUMO, Gln-ThrGly-Gly. On the other hand, the Leu-Arg-Gly-Gly sequence found in ubiquitin and Nedd8 was found to be the optimal one for SENP8 and, interestingly, for SENPs 6 and 7 [58]. However, analysis of natural substrates containing this sequence (ubiquitin, Nedd8 and ISG15) revealed that they are not cleaved by SENPs 6 and 7; moreover, both these SENPs undergo substrate activation by truncated SUMO2 moieties [25]. This is especially intriguing in the light of later reports demonstrating that they are able to process SUMO, in the form of polySUMO chains, however. There are two reasonable explanations for the observed 'false' substrate specificity. First, SENPs 6 and 7 could perhaps cleave yet-unidentified substrates, or the altered specificity actually hinders them from cleaving monoSUMOylated proteins and directs their activity towards polySUMO chains by additional interactions. However, structure-based mutations in the catalytic domain of SENP7 that disrupt its ability to recognize multiple SUMO molecules had no effect on its ability to cleave SUMO chains [62]. The altered specificities of SENPs 6 and 7 could even be perceived as an evolutionary delay in changes affecting their active sites. A recent report on a SENP found in P. falciparum seems to partially explain this phenomenon. Despite being a typical SUMO protease, $P f$-SENP1 recognizes both peptidic sequences, Gln-Thr-Gly-Gly and Leu-Arg-Gly-Gly, equally well [53]. In addition, SENPs 1-3 and 5 cluster phylogenetically with yeast Ulp1 [63] which cleaves the yeast SUMO (Smt3), while SENPs 6 and 7 cluster with cluster Ulp2 which has been implicated in the processing of polySUMO chains [64].

For the bacterial SUMO protease XopD, as there are no SUMO homologs in bacteria, its specificity has evolved to recognize plant SUMO molecules [54], a fascinating coevolution phenomenon of a host and a pathogen, in our opinion.

\section{The Influence of Pathogens on the Host SUMOylation Machinery}

There are numerous virus-encoded ubiquitin-like proteases that exhibit deubiquitinating activity when expressed in host cells $[10,65]$. Recently, an elegant study reported the discovery of a Nedd8-specific protease from the Epstein-Barr virus, BPLF1, which deneddylates host cullins and influences viral DNA replication [66]. Addi- tionally, ElaD from Escherichia coli has been shown to have deubiquitinating activity [67], and DUBs that process both ubiquitin and Nedd 8 have been found in pathogenic bacteria, such as Chlamydia trachomatis [68].

\section{Xanthomonas Outer Protein D - A Bacterial Protease Acting on SUMOylated Plant Proteins}

As of yet, no SUMO-specific protease has been reported to be found in viruses. However, one of the first SUMO-specific protease structures to be solved was of Xanthomonas outer protein D (XopD) [69], which is a SUMO-specific cysteine protease from the pathogen $X$. campestris pathovar vesicatoria [54], which is responsible for the bacterial spot disease on tomato and pepper plants [70]. XopD is a bacterial virulence factor that is secreted directly into the cytosol of plant cells by the Xcv type 3 secretion system (TTSS) [71], which is essential for bacterial virulence and is responsible for the delivery of numerous bacterial effector proteins into host plant cells [72]. In planta, XopD translocates to the nucleus and deSUMOylates host plant proteins [54], presumably contributing to the virulence of the pathogen by turning off plant response mechanisms (see fig. 2). The nuclear localization of XopD, for example, suggests that its substrate repertoire would be nuclear SUMO-conjugated proteins. Interestingly, it has also been shown that XopD can heterodimerize with host transcription factors, thereby directly affecting plant transcription [73], further making the case for the targets of XopD to reside within the nuclei of host cells. As for resident SENPs in plants, there have been 5 deSUMOylating enzymes identified in Arabidopsis thaliana thus far [74]. Arabidopsis SENPs have been characterized in vitro and have been shown to cleave plant and mammalian SUMO molecules as well [75]. It is still unclear what the exact substrates of a pathogenic SUMO protease could be, that host enzymes in plants do not or cannot cleave. However, since all human SENPs possess the intrisinc ability to cleave SUMO chains [29], it is highly probable that XopD would also be able to remove polySUMO from conjugated proteins, although such activity for XopD has yet to be formally demonstrated. In addition, an extended N-terminal region of XopD has been suggested to be crucial for its virulence [76], which is intriguing, as it is one of the few functional characterizations of the N-terminal domain of a SUMO protease. Another study, in the case of Ulp1, has demonstrated the involvement of its $\mathrm{N}$-terminus in restricting the specificity of Ulp1 to a subset of SUMOylated proteins in 


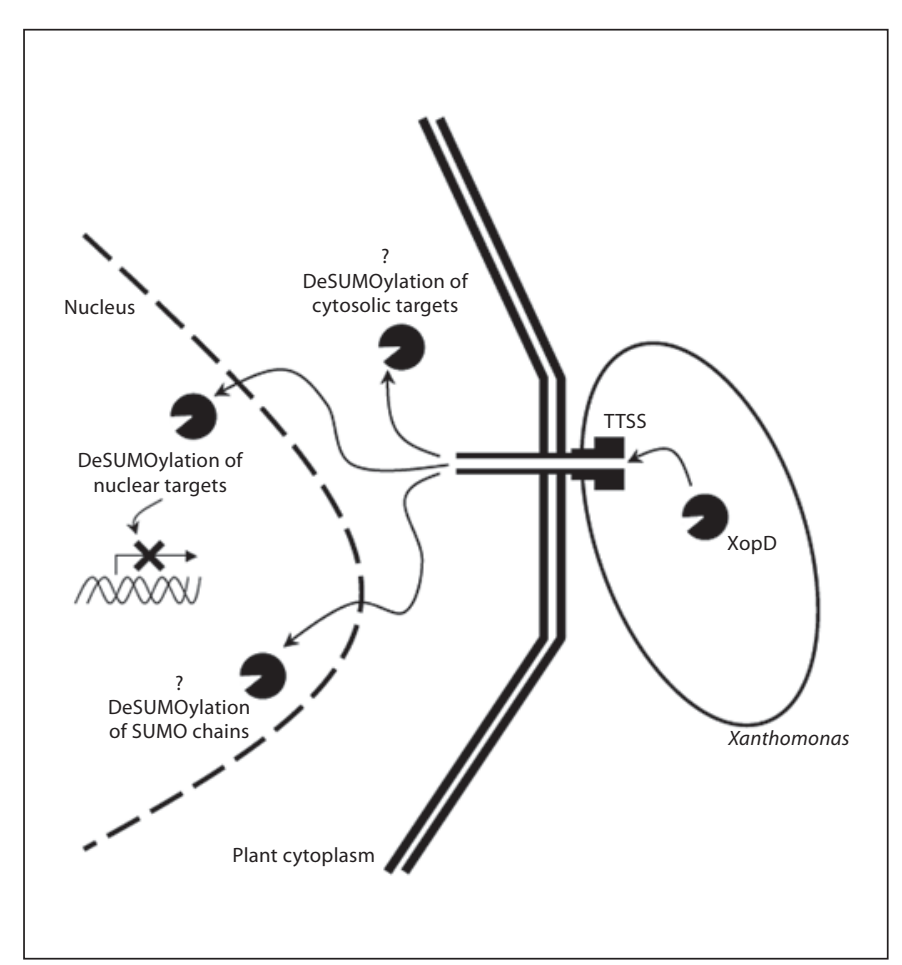

Fig. 2. The functions of XopD in bacterial infection of plant cells: the injection of XopD into host plant cells by the TTSS of X. campestris pathovar vesicatoria. Upon delivery, XopD translocates to the nucleus and deSUMOylates nuclear SUMOylated proteins, presumably turning off the transcription of genes required for plant defense mechanisms. It is currently unclear if XopD performs additional deSUMOylating activities in the plant cytosol or whether XopD can cleave SUMO chains.

yeast [51]. Mammalian SENPs also possess significant $\mathrm{N}$ terminal domains, which appear to be largely unstructured and without homology to any known domain. They have been vaguely suggested to be important for substrate specificity and subcellular localization [63]; however, more investigation is warranted in this regard.

Recently, another TTSS bacterial virulence factor from the YopJ family, AvrXv4 [77], has been shown to reduce the levels of SUMOylated protein in host plant cells upon infection [78]; however, in vitro AvrXv4 was inactive towards SUMO conjugates.

\section{Gam1 - A Viral Inhibitor of Host SUMOylation}

Within the realm of modifications by SUMO, pathogens have ample opportunity to interfere with host proteins by inhibiting or enhancing their SUMOylation, or

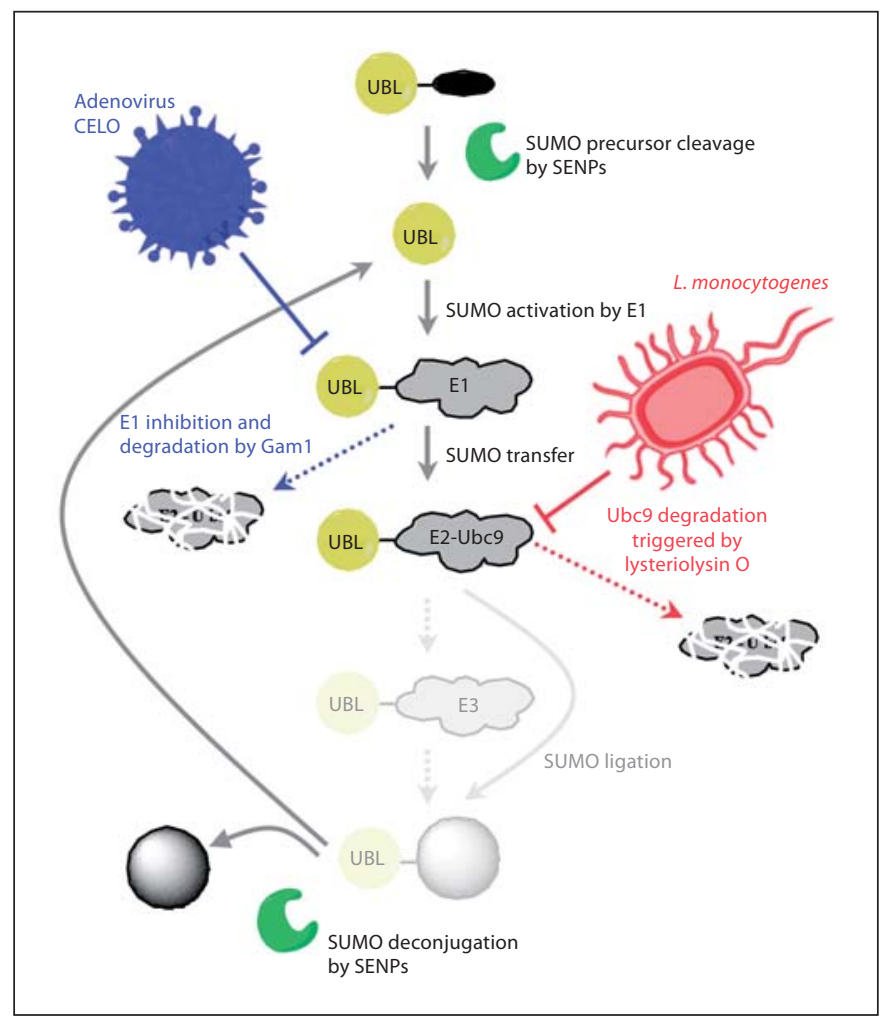

Fig. 3. The influence of pathogens on the host SUMOylation pathway: examples of a viral protein [Gam1 from adenovirus CELO (in blue)] and a bacterial virulence factor [lysteriolysin $\mathrm{O}$ from $L$. monocytogenes (in pink)] influencing the SUMO pathway of host cells upon infection, resulting in global hypoSUMOylation of target proteins.

by using their own proteins as a target for SUMOylation by the host conjugating machinery. This latter phenomenon has been documented for the first time for viruses, which used their own proteins as substrates for modification by host SUMO proteins [79]. Additionally, Boggio et al. [46] reported that an adenoviral protein (Gaml), which is a strong and global transcriptional activator of cellular and viral genes, exhibited inhibitory activity towards HDAC1, a deacetylating enzyme, as well as towards the SUMO pathway of host cells. Upon Gam1 binding to the SUMO1 E1 enzyme (see fig. 3), the enzymatic activity of the E1 was inhibited, leading to the direct or indirect degradation of the E1-Gaml complex, as well as to the degradation of the SUMO E2, by yet undefined mechanisms. This resulted in the net accumulation of SUMO-unmodified substrates, while in parallel, it was also demonstrated that Gam1 had no influence on SENP inhibition, either in vitro or in vivo. Interestingly, the in- 
volvement of the ubiquitin/proteasome pathway had also been implicated due the fact that the stability of the E1 heterodimer was not affected at the transcriptional level, but at the protein level. Furthermore, treatment of cells with proteasome inhibitors seems to have completely blocked the Gam1-mediated degradation of the SUMO E1 and E2 enzymes. Unfortunately, despite determination of the influence of Gam1 on the SUMO pathway, its biological consequences during viral infection are not clear and require further investigation.

\section{Listeriolysin 0 - A Bacterial Inhibitor of Global Host SUMO Conjugation}

As for the influence of pathogenic bacteria on host SUMOylation, a recent work by Ribet et al. [47] demonstrate that Listeria monocytogenes has substantial influence on the host SUMOylation machinery during its infection. This intracellular, foodborne pathogen is a causative agent of listeriosis, which results in $20-30 \%$ deaths once patients have become infected. During infection, LLO, a pore-forming toxin from the bacteria, induces the specific degradation of Ubc9 (see fig. 3). Due to the fact that Ubc9 is the only E2 enzyme in the human SUMOylation pathway, this results in a complete inhibition of host protein SUMO modification and thus results in global cellular deSUMOylation. Presumably, it helps bacteria to impair the activity of several important host proteins and aids in facilitating the replication of L. monocytogenes. The importance of this observation was confirmed in elegant experiments, where SUMO1 and SUMO2 were overexpressed in HeLa cells, which were then infected with L. monocytogenes. The SUMOylated protein levels in these infected cells were significantly reduced compared to the uninfected control. This was further confirmed in a mouse-model experiment, where C57B1/6J mice were infected with L. monocytogenes bacteria and examined for the changes in Ubc9 levels after 48 and $72 \mathrm{~h}$; the levels were reduced. However, the reduction of the E2 enzyme levels, induced by listeriolysin O, was shown to be in a proteasome-independent fashion, both in vivo and in cultured cells. Nevertheless, the exact mechanism of LLO-mediated degradation of Ubc9 is still to be determined. Similar effects were also observed for other pore-forming toxins found in other bacteria, perfringolysin $\mathrm{O}$ and pneumolysin. This raises the possibility that global inhibition of SUMO conjugation is a universal strategy utilized by pathogenic bacteria to aid in the infection of host cells. Coincidentally, these results mirror the effect of XopD on host cells (see above), which has been shown to cause global host (plant) cell deSUMOylation. So it seems that tipping the host SUMOylation balance could serve to benefit bacterial infection, most probably in combination with the effects of other bacterial virulence factors.

\section{Influence of Pathogens on Other Ubiquitin-Like Modifiers}

Certain bacteria can also affect the host Nedd8-SENP8 system. It has been shown that the interaction of viable commensal bacteria with host intestinal epithelial cells results in a robust production of reactive oxygen species, which in turn can influence the neddylation of Cullin-1, an essential component of the SCF (SKP1-CUL1-F-box protein) E3 ligase complex. It is conceivable then, as has been shown before, that the lack of neddylation of this complex could result in the inhibition of the NF- $\kappa \mathrm{B}$ pathway, which could be beneficial for the bacteria [80]. Interestingly, this scenario is somewhat similar to the case of a deneddylating enzyme expressed by the Epstein-Barr virus, which also leads to host cullin-deneddylation, aiding in the replication of the virus [66]. These processes underscore the sensitivity of cullins to minor shifts in their neddylation status, which is ruthlessly exploited by pathogens.

In addition, a viral example for host UBL system interference is the influenza B virus NS1 protein, which inhibits conjugation of another UBL which is induced by interferon-alpha/beta - ISG15 [81]. In this case, a specific region of the viral NS1B protein inhibits covalent conjugation of ISG15 to target proteins by its influence on the first ISG15 conjugation step catalyzed by UBE1L, which is the E1 enzyme for ISG15 [82, 83]. Also in this case, it is hard to say how the conjugation of ISG15 modulates the replication of human influenza B virus and what the real biological consequences of this process are. However, as conjugation by ISG15 has recently been shown to mark newly synthesized proteins for degradation as part of an antiviral response [84], it is conceivable that NS1B counteracts ISG15 conjugation and thus the degradation of de novo viral proteins to ensure efficient viral replication.

\section{Conclusions}

PTMs are key players in virtually all stages of cell development and function. Among them, SUMOylation has recently gained much interest due to the scale of this 
Table 1. Summary of pathogens and their effector proteins influencing host UBL dynamics

\begin{tabular}{|c|c|c|c|c|}
\hline $\begin{array}{l}\text { UBL } \\
\text { pathway }\end{array}$ & $\begin{array}{l}\text { Pathogen } \\
\text { species }\end{array}$ & $\begin{array}{l}\text { Virulence factor/ } \\
\text { effector protein }\end{array}$ & Cellular consequences in the host organism & References \\
\hline SUMO & X. campestris & $\begin{array}{l}\text { XopD } \\
\text { (a SUMO protease) }\end{array}$ & $\begin{array}{l}\text { Injection of XopD into the cytosol of host plant cells via a TTSS } \\
\text { causes deSUMOylation of host plant proteins in the nucleus, } \\
\text { turning off genes involved in plant defense mechanisms }\end{array}$ & {$[54,69]$} \\
\hline SUMO & $\begin{array}{l}\text { Adenovirus } \\
\text { CELO }\end{array}$ & $\begin{array}{l}\text { Gam1 } \\
\text { (early viral gene } \\
\text { product) }\end{array}$ & $\begin{array}{l}\text { The interaction of Gam } 1 \text { with the E1 SUMO-activating enzyme } \\
\text { results in E1 inhibition and subsequent degradation of both the } \\
\text { SUMO E1 and E2 enzymes, by yet undefined mechanisms, } \\
\text { resulting in global hypoSUMOylation }\end{array}$ & {$[46,79]$} \\
\hline SUMO & L. monocytogenes & $\begin{array}{l}\text { LLO } \\
\text { (pore-forming } \\
\text { toxin) }\end{array}$ & $\begin{array}{l}\text { During infection by L. monocytogenes, LLO causes the specific } \\
\text { degradation of Ubc9, the sole SUMO E2 enzyme, by a yet } \\
\text { undefined mechanism, resulting in global host hypoSUMOylation }\end{array}$ & {$[16,47]$} \\
\hline Nedd8 & $\begin{array}{l}\text { Epstein-Barr } \\
\text { virus }\end{array}$ & $\begin{array}{l}\text { BPLF1 } \\
\text { (a Nedd8 protease) }\end{array}$ & $\begin{array}{l}\text { BPLF1 from Epstein-Barr virus destabilizes host cullins by its } \\
\text { deneddylating activity, leading to the stabilization of CDT1 to } \\
\text { allow viral DNA replication. }\end{array}$ & {$[66]$} \\
\hline ISG15 & $\begin{array}{l}\text { Influenza B } \\
\text { virus }\end{array}$ & $\begin{array}{l}\text { NS1B } \\
\text { (nonstructural } \\
\text { viral protein) }\end{array}$ & $\begin{array}{l}\text { Upon viral infection, ISG15 expression and conjugation is induced } \\
\text { as part of the antiviral response; however, NS1B inhibits ISG15 } \\
\text { conjugation by inhibiting UBE1L, the E1 enzyme for ISG15. }\end{array}$ & {$[81]$} \\
\hline
\end{tabular}

cellular phenomena and its wide implication in many pathological disorders, like cancer or neurological diseases. While the SUMO system in humans is relatively well described, on both the conjugation and deconjugation fronts, as well as in ideal homeostatis and during pathological conditions, there are still big gaps to fill with regard to how the human SUMO system could be exploited during viral or bacterial infections. Information has recently started to emerge, yet still little is known about the SUMO (or SUMO-like) systems of pathogens or about the influence pathogens can exert via various virulence factors on the SUMO system of host cells during infections. The recent discoveries presented in this review (see table 1) highlight the importance of this phenomenon, which suggests that pathogens could easily be capable of influencing the host SUMO system in humans as well. These studies open the field wide for further investigations and may even suggest that such an avenue could be a fruitful path to take for developing therapeutics that could limit bacterial or viral infections, such as by interfering with the way bacteria and viruses interfere with host SUMO systems.

A common theme for the battle of the host SUMO system versus pathogens emerges from the discoveries mentioned in this review. The message is that - regardless of whether it is a plant pathogen expressing an exogenous enzyme and delivering it to host plant cells to perform its function, or it is a human disease causing bacteria that induces the degradation of a key enzyme in the SUMO system - the outcome is identical i.e. the global hypoSUMOylation of host SUMOylated proteins upon viral and bacterial infections. The picture that is taking shape is that the deSUMOylation of host proteins is beneficial for pathogens, so that the inhibition of host deSUMOylating enzymes could also be a viable strategy to boost human defense mechanisms. This is intriguing, exciting and challenging, as currently there are no known good deSUMOylating enzyme inhibitors. To us, it seems like the race is on again to find ways to deal with hypoSUMOylation once Trojan horse pathogenic contents have been released in host cells.

\section{Acknowledgements}

M.D.'s laboratory was supported by the Foundation for Polish Science and the State Scientific Research Grant N-N401-042838. M.B. was supported by an NCI Molecular Oncology and Immunology Training Grant Fellowship (2T32CA009161-36A1). He wishes to acknowledge the support of Tony T. Huang, in whose laboratory at NYU he is currently a post-doctoral fellow. 


\section{References}

-1 Kerscher O, Felberbaum R, Hochstrasser M: Modification of proteins by ubiquitin and ubiquitin-like proteins. Annu Rev Cell Dev Biol 2006;22:159-180.

\2 Pearce MJ, Mintseris J, Ferreyra J, Gygi SP, Darwin KH: Ubiquitin-like protein involved in the proteasome pathway of mycobacterium tuberculosis. Science 2008;322:11041107.

- 3 Hunter T: Protein kinases and phosphatases: the yin and yang of protein phosphorylation and signaling. Cell 1995;80:225-236.

$\checkmark 4$ King CM, Glowinski IB: Acetylation, deacetylation and acyltransfer. Environ Health Perspect 1983;49:43-50.

5 Ruddock LW, Molinari M: N-glycan processing in ER quality control. J Cell Sci 2006; 119:4373-4380.

-6 Reyes-Turcu FE, Ventii KH, Wilkinson KD: Regulation and cellular roles of ubiquitinspecific deubiquitinating enzymes. Annu Rev Biochem 2009;78:363-397.

7 Johnson ES: Protein modification by SUMO. Annu Rev Biochem 2004;73:355-382.

$\checkmark 8$ Mukhopadhyay D, Dasso M: Modification in reverse: the SUMO proteases. Trends Biochem Sci 2007;32:286-295.

-9 Imkamp F, Striebel F, Sutter M, Ozcelik D, Zimmermann N, Sander P, Weber-Ban E: Dop functions as a depupylase in the prokaryotic ubiquitin-like modification pathway. EMBO Rep 2010;11:791-797.

$\checkmark 10$ Isaacson MK, Ploegh HL: Ubiquitination, ubiquitin-like modifiers, and deubiquitination in viral infection. Cell Host Microbe 2009;5:559-570.

11 Randow F, Lehner PJ: Viral avoidance and exploitation of the ubiquitin system. Nat Cell Biol 2009;11:527-534.

12 Jacob T, Van den Broeke C, Favoreel HW: Viral serine/threonine protein kinases. J Viro 2011;85:1158-1173.

13 Munter S, Way M, Frischknecht F: Signaling during pathogen infection. Sci STKE 2006; 2006:re5.

14 Best SM: Viral subversion of apoptotic enzymes: escape from death row. Annu Rev Microbiol 2008;62:171-192.

15 Bird PI: Serpins and regulation of cell death. Results Probl Cell Differ 1998;24:63-89.

16 Ribet D, Cossart P: Post-translational modifications in host cells during bacterial infection. FEBS Lett 2010;584:2748-2758.

- 17 Mahajan R, Delphin C, Guan T, Gerace L, Melchior F: A small ubiquitin-related polypeptide involved in targeting RanGAP1 to nuclear pore complex protein RanBP2. Cell 1997;88:97-107.

18 Gill G: Something about SUMO inhibits transcription. Curr Opin Genet Dev 2005; 15:536-541.
19 Panse VG, Kressler D, Pauli A, Petfalski E, Gnadig M, Tollervey D, Hurt E: Formation and nuclear export of preribosomes are functionally linked to the small-ubiquitinrelated modifier pathway. Traffic 2006; 7 1311-1321.

20 Martin S, Nishimune A, Mellor JR, Henley JM: SUMOylation regulates kainate-receptor-mediated synaptic transmission. Nature 2007;447:321-325.

21 Dasso M: Emerging roles of the SUMO pathway in mitosis. Cell Div 2008;3:5

22 Bergink S, Jentsch S: Principles of ubiquitin and SUMO modifications in DNA repair. Nature 2009;458:461-467.

23 Zhao X, Blobel G: A SUMO ligase is part of a nuclear multiprotein complex that affects DNA repair and chromosomal organization. Proc Natl Acad Sci USA 2005; 102:47774782.

24 Geiss-Friedlander R, Melchior F: Concepts in SUMOylation: a decade on. Nat Rev Mol Cell Biol 2007;8:947-956.

25 Mikolajczyk J, Drag M, Békés M, Cao JT, Ronai Z, Salvesen GS: Small ubiquitin-related modifier (SUMO)-specific proteases: profiling the specificities and activities of human SENPs. J Biol Chem 2007;282:26217-26224.

26 Zhu S, Goeres J, Sixt KM, Békés M, Zhang $\mathrm{XD}$, Salvesen GS, Matunis MJ: Protection from isopeptidase-mediated deconjugation regulates paralog-selective SUMOylation of RanGAP1. Mol Cell 2009;33:570-580.

27 Matic I, van Hagen M, Schimmel J, Macek B, Ogg SC, Tatham MH, Hay RT, Lamond AI, Mann M, Vertegaal AC: In vivo identification of human small ubiquitin-like modifier polymerization sites by high accuracy mass spectrometry and an in vitro to in vivo strategy. Mol Cell Proteomics 2008;7:132-144.

28 Tatham MH, Jaffray E, Vaughan OA, Desterro JM, Botting $\mathrm{CH}$, Naismith JH, Hay RT: Polymeric chains of SUMO-2 and SUMO-3 are conjugated to protein substrates by SAE1/SAE2 and Ubc9. J Biol Chem 2001;276: 35368-35374.

-29 Békés M, Prudden J, Srikumar T, Raught B, Boddy MN, Salvesen GS: The dynamics and mechanism of SUMO chain deconjugation by SUMO-specific proteases. J Biol Chem 2011;286:10238-10247.

30 Golebiowski F, Matic I, Tatham MH, Cole C, Yin Y, Nakamura A, Cox J, Barton GJ, Mann M, Hay RT: System-wide changes to SUMO modifications in response to heat shock. Sci Signal 2009;2:ra24.

-31 Tatham MH, Matic I, Mann M, Hay RT: Comparative proteomic analysis identifies a role for SUMO in protein quality control. Sci Signal 2011;4:rs4.

- 32 Perry JJ, Tainer JA, Boddy MN: A simultaneous role for SUMO and ubiquitin. Trends Biochem Sci 2008;33:201-208.
33 Prudden J, Pebernard S, Raffa G, Slavin DA, Perry JJ, Tainer JA, McGowan CH, Boddy $\mathrm{MN}$ : SUMO-targeted ubiquitin ligases in genome stability. EMBO J 2007;26:4089-4101.

-34 Sun H, Leverson JD, Hunter T: Conserved function of RNF4 family proteins in eukaryotes: targeting a ubiquitin ligase to SUMOylated proteins. EMBO J 2007;26:41024112.

-35 Drag M, Salvesen GS: DeSUMOylating enzymes - SENPs. IUBMB Life 2008;60:734742 .

36 Hay RT: SUMO: a history of modification. Mol Cell 2005;18:1-12.

- 37 Matic I, Schimmel J, Hendriks IA, van Santen MA, van de Rijke F, van Dam H, Gnad F, Mann M, Vertegaal AC: Site-specific identification of SUMO-2 targets in cells reveals an inverted SUMOylation motif and a hydrophobic cluster SUMOylation motif. Mol Cell 2010;39:641-652.

- 38 Lois LM, Lima CD: Structures of the SUMO E1 provide mechanistic insights into SUMO activation and E2 recruitment to E1. EMBO J 2005;24:439-451.

- 39 Gareau JR, Lima CD: The sumo pathway: emerging mechanisms that shape specificity, conjugation and recognition. Nat Rev Mol Cell Biol 2010;11:861-871.

40 Olsen SK, Capili AD, Lu X, Tan DS, Lima CD: Active site remodelling accompanies thioester bond formation in the SUMO E1. Nature 2010;463:906-912.

41 Johnson ES, Blobel G: Ubc9p is the conjugating enzyme for the ubiquitin-like protein Smt3p. J Biol Chem 1997;272:26799-26802.

42 Reverter D, Lima CD: Insights into E3 ligase activity revealed by a SUMO-RanGAP1Ubc9-Nup358 complex. Nature 2005;435: 687-692.

43 Palvimo JJ: PIAS proteins as regulators of small ubiquitin-related modifier (SUMO) modifications and transcription. Biochem Soc Trans 2007;35:1405-1408.

44 Pichler A, Knipscheer P, Oberhofer E, van Dijk WJ, Korner R, Olsen JV, Jentsch S, Melchior F, Sixma TK: SUMO modification of the ubiquitin-conjugating enzyme E2-25K. Nat Struct Mol Biol 2005;12:264-269.

-45 Petroski MD, Deshaies RJ: Function and regulation of cullin-ring ubiquitin ligases. Nat Rev Mol Cell Biol 2005;6:9-20.

46 Boggio R, Colombo R, Hay RT, Draetta GF, Chiocca S: A mechanism for inhibiting the SUMO pathway. Mol Cell 2004;16:549-561.

-47 Ribet D, Hamon M, Gouin E, Nahori MA, Impens F, Neyret-Kahn H, Gevaert K, Vandekerckhove J, Dejean A, Cossart P: Listeria monocytogenes impairs SUMOylation for efficient infection. Nature 2010;464: 1192-1195. 
48 Wilkinson KD: Regulation of ubiquitin-dependent processes by deubiquitinating enzymes. FASEB J 1997;11:1245-1256.

-49 Rawlings ND, Barrett AJ, Bateman A: MEROPS: the peptidase database. Nucleic Acids Res 2010;38:D227-D233.

-50 Li SJ, Hochstrasser M: The yeast Ulp2 (Smt4) gene encodes a novel protease specific for the ubiquitin-like Smt3 protein. Mol Cell Biol 2000;20:2367-2377.

- 51 Li SJ, Hochstrasser M: The Ulp1 SUMO isopeptidase: distinct domains required for viability, nuclear envelope localization, and substrate specificity. J Cell Biol 2003;160: 1069-1081.

- 52 Li SJ, Hochstrasser M: A new protease required for cell-cycle progression in yeast. Nature 1999;398:246-251.

- 53 Ponder EL, Albrow VE, Leader BA, Békés M, Mikolajczyk J, Fonovic UP, Shen A, Drag M, Xiao J, Deu E, Campbell AJ, Powers JC, Salvesen GS, Bogyo M: Functional characterization of a SUMO deconjugating protease of Plasmodium falciparum using newly identified small molecule inhibitors. Chem Biol 2011;18:711-721.

-54 Hotson A, Chosed R, Shu H, Orth K, Mudgett MB: Xanthomonas type III effector Xopd targets SUMO-conjugated proteins in planta. Mol Microbiol 2003;50:377-389.

-55 Shen LN, Liu H, Dong C, Xirodimas D, Naismith JH, Hay RT: Structural basis of Nedd8 ubiquitin discrimination by the deneddylating enzyme Nedp1. EMBO J 2005;24:13411351.

56 Békés M, Salvesen GS: Mechanisms and substrate specificity of deSUMOylating enyzmes; in Di Napoli M, Wojcik C (eds): The Ubiquitin Proteasome System in the Central Nervous System: From Physiology to Pathology - 2008 update. New York, Nova Science, 2010, pp 5-32.

-57 Yeh ET: SUMOylation and de-SUMOylation: wrestling with life's processes. J Biol Chem 2009;284:8223-8227.

- 58 Drag M, Mikolajczyk J, Krishnakumar IM, Huang Z, Salvesen GS: Activity profiling of human deSUMOylating enzymes (SENPs) with synthetic substrates suggests an unexpected specificity of two newly characterized members of the family. Biochem J 2008;409: 461-469.

-59 Reverter D, Lima CD: Structural basis for SENP2 protease interactions with SUMO precursors and conjugated substrates. Nat Struct Mol Biol 2006;13:1060-1068.
60 Shen L, Tatham MH, Dong C, Zagorska A, Naismith JH, Hay RT: SUMO protease SENP1 induces isomerization of the scissile peptide bond. Nat Struct Mol Biol 2006;13: 1069-1077.

61 Komander D, Clague MJ, Urbe S: Breaking the chains: structure and function of the deubiquitinases. Nat Rev Mol Cell Biol 2009; 10:550-563.

62 Lima CD, Reverter D: Structure of the human SENP7 catalytic domain and poly-SUMO deconjugation activities for SENP6 and SENP7. J Biol Chem 2008;283:32045-32055.

63 Hay RT: SUMO-specific proteases: a twist in the tail. Trends Cell Biol 2007;17:370-376.

64 Bylebyl GR, Belichenko I, Johnson ES: The SUMO isopeptidase Ulp2 prevents accumulation of SUMO chains in yeast. J Biol Chem 2003;278:44113-44120.

65 Sompallae R, Gastaldello S, Hildebrand S, Zinin N, Hassink G, Lindsten K, Haas J, Persson B, Masucci MG: Epstein-Barr virus encodes three bona fide ubiquitin-specific proteases. J Virol 2008;82:10477-10486.

-66 Gastaldello S, Hildebrand S, Faridani O, Callegari S, Palmkvist M, Di Guglielmo C, Masucci MG: A deneddylase encoded by Epstein-Barr virus promotes viral DNA replication by regulating the activity of cullinring ligases. Nat Cell Biol 2010;12:351-361.

67 Catic A, Misaghi S, Korbel GA, Ploegh HL: ElaD, a deubiquitinating protease expressed by E. coli. PLoS One 2007;2:e381.

68 Misaghi S, Balsara ZR, Catic A, Spooner E, Ploegh HL, Starnbach MN: Chlamydia trachomatis-derived deubiquitinating enzymes in mammalian cells during infection. Mol Microbiol 2006;61:142-150.

69 Chosed R, Tomchick DR, Brautigam CA, Mukherjee S, Negi VS, Machius M, Orth K: Structural analysis of Xanthomonas Xopd provides insights into substrate specificity of ubiquitin-like protein proteases. J Biol Chem 2007;282:6773-6782.

70 Jones JB, Stall RE, Bouzar H: Diversity among xanthomonads pathogenic on pepper and tomato. Annu Rev Phytopathol 1998;36: 41-58.

71 Noel L, Thieme F, Nennstiel D, Bonas U: Two novel type III-secreted proteins of Xanthomonas campestris pv. vesicatoria are encoded within the hrp pathogenicity island. J Bacteriol 2002;184:1340-1348.

72 Buttner D, Bonas U: Getting across - bacterial type III effector proteins on their way to the plant cell. EMBO J 2002;21:5313-5322.

-73 Kim JG, Taylor KW, Hotson A, Keegan M, Schmelz EA, Mudgett MB: Xopd SUMO protease affects host transcription, promotes pathogen growth, and delays symptom development in Xanthomonas-infected tomato leaves. Plant Cell 2008;20:1915-1929.
74 Murtas G, Reeves PH, Fu YF, Bancroft I, Dean C, Coupland G: A nuclear protease required for flowering-time regulation in arabidopsis reduces the abundance of small ubiquitin-related modifier conjugates. Plant Cell 2003; 15:2308-2319.

75 Chosed R, Mukherjee S, Lois LM, Orth K: Evolution of a signalling system that incorporates both redundancy and diversity: arabidopsis SUMOylation. Biochem J 2006;398: 521-529.

76 Canonne J, Marino D, Noel LD, Arechaga I, Pichereaux C, Rossignol M, Roby D, Rivas S: Detection and functional characterization of a 215 amino acid $\mathrm{N}$-terminal extension in the Xanthomonas type III effector Xopd. PLoS One 2010;5:e15773.

77 Gurlebeck D, Thieme F, Bonas U: Type III effector proteins from the plant pathogen Xanthomonas and their role in the interaction with the host plant. J Plant Physiol 2006; 163:233-255.

78 Roden J, Eardley L, Hotson A, Cao Y, Mudgett MB: Characterization of the Xanthomonas AvrXv4 effector, a SUMO protease translocated into plant cells. Mol Plant Microbe Interact 2004;17:633-643.

79 Boggio R, Chiocca S: Viruses and SUMOylation: recent highlights. Curr Opin Microbiol 2006;9:430-436.

$>80$ Collier-Hyams LS, Sloane V, Batten BC, Neish AS: Cutting edge: bacterial modulation of epithelial signaling via changes in neddylation of cullin-1. J Immunol 2005; 175: 4194-4198.

-81 Yuan W, Krug RM: Influenza B virus NS1 protein inhibits conjugation of the interferon (IFN)-induced ubiquitin-like ISG15 protein. EMBO J 2001;20:362-371.

82 Chang YG, Yan XZ, Xie YY, Gao XC, Song AX, Zhang DE, Hu HY: Different roles for two ubiquitin-like domains of ISG15 in protein modification. J Biol Chem 2008;283: 13370-13377.

83 Li L, Wang D, Jiang Y, Sun J, Zhang S, Chen Y, Wang X: Crystal structure of human ISG15 in complex with influenza B virus NS1B. J Biol Chem 2011;286:30258-30262.

84 Durfee LA, Lyon N, Seo K, Huibregtse JM: The ISG15 conjugation system broadly targets newly synthesized proteins: implications for the antiviral function of ISG15. Mol Cell 2010;38:722-732. 